\title{
Generalized Manning Condensation Model Captures the RNA Ion Atmosphere
}

\author{
Ryan L. Hayes, ${ }^{1}$ Jeffrey K. Noel, ${ }^{1}$ Ana Mandic, ${ }^{2}$ Paul C. Whitford, ${ }^{3}$ Karissa Y. Sanbonmatsu, ${ }^{4}$ \\ Udayan Mohanty, ${ }^{5}$ and José N. Onuchic ${ }^{1, *}$ \\ ${ }^{1}$ Center for Theoretical Biological Physics and Department of Physics and Astronomy, Rice University, Houston, Texas 77030, USA \\ ${ }^{2}$ Department of Biomedical Engineering, University of Houston, Houston, Texas 77004, USA \\ ${ }^{3}$ Department of Physics, Northeastern University, Boston, Massachusetts 02115, USA \\ ${ }^{4}$ Theoretic Biology and Biophysics, Theoretic Division, Los Alamos National Labs, Los Alamos, New Mexico 87545, USA \\ ${ }^{5}$ Department of Chemistry, Boston College, Chestnut Hill, Massachusetts 02467, USA
}

(Received 27 February 2015; published 26 June 2015)

\begin{abstract}
RNA is highly sensitive to the ionic environment and typically requires $\mathrm{Mg}^{2+}$ to form compact structures. There is a need for models capable of describing the ion atmosphere surrounding RNA with quantitative accuracy. We present a model of RNA electrostatics and apply it within coarse-grained molecular dynamics simulation. The model treats $\mathrm{Mg}^{2+}$ ions explicitly to account for ion-ion correlations neglected by mean-field theories. Since mean-field theories capture $\mathrm{KCl}$ well, it is treated implicitly by a generalized Manning counterion condensation model. The model extends Manning condensation to deal with arbitrary RNA conformations, nonlimiting $\mathrm{KCl}$ concentrations, and the ion inaccessible volume of RNA. The model is tested against experimental measurements of the excess $\mathrm{Mg}^{2+}$ associated with the RNA, $\Gamma_{2+}$, because $\Gamma_{2+}$ is directly related to the $\mathrm{Mg}^{2+}$-RNA interaction free energy. The excellent agreement with experiment demonstrates that the model captures the ionic dependence of the RNA free energy landscape.
\end{abstract}

RNA is sensitive to the ionic environment because it is strongly negatively charged and yet frequently folds into compact configurations. Such compact configurations require positive counterions to balance the RNA charge. $\mathrm{Mg}^{2+}$ is especially effective in stabilizing compact configurations, as most RNA tertiary structure will not form in the absence of $\mathrm{Mg}^{2+}$ [1]. Simplified or coarse-grained molecular dynamics simulations are an ideal tool for studying the molecular details of slow processes in RNA [2-6]; however, their accuracy is limited at present by the lack of accurate and computationally efficient descriptions of the atmosphere of ions associated with RNA. We generalize the theory of Manning counterion condensation [7] to arbitrary geometries and concentrations, making it applicable to compact RNA structures, and show that this model accurately represents the ion atmosphere around RNA.

The ubiquity of $\mathrm{Mg}^{2+}$ in RNA structure and dynamics arises because $\mathrm{Mg}^{2+}$ is small and divalent. The small size of $\mathrm{Mg}^{2+}$ allows it to interact more closely with RNA than larger ions do [8,9]. Because $\mathrm{Mg}^{2+}$ is divalent, only half as many $\mathrm{Mg}^{2+}$ as monovalent ions must be localized around RNA to balance its charge, allowing twice the entropic cost to be paid per ion $[7,10]$. Consequently, $\mathrm{Mg}^{2+}$ can outcompete monovalent ions present at much higher concentrations to associate with RNA. The divalence of $\mathrm{Mg}^{2+}$ also allows it to induce effective attraction between otherwise repulsive phosphates [10-12]. As a result, $\mathrm{Mg}^{2+}$ strongly favors compact RNA conformations [10], and it can slow kinetics by raising the free energy of less compact transition states [13]. In many cases, changing the $\mathrm{Mg}^{2+}$ concentration can switch stability between two conformational basins [14-17]. Electrostatic models capable of describing $\mathrm{Mg}^{2+}$-RNA interactions are needed to connect with these experiments and to describe the RNA energy landscape.

The simplest model of electrostatics in ionic solutions is Debye-Hückel electrostatics, in which the ion density is given by the linearized Boltzmann distribution, and dielectric heterogeneity and ion accessibility are neglected. Coarsegrained models of RNA have used a Debye-Hückel treatment of $\mathrm{KCl}[18,19]$. Such a treatment is not ideal for $\mathrm{Mg}^{2+}$ because the linearized Boltzmann distribution is a poor approximation for strong $\mathrm{Mg}^{2+}$-RNA interactions near RNA. In addition, Debye-Hückel is unable to produce the effective attraction between phosphates that $\mathrm{Mg}^{2+}$ can induce.

Nonlinear Poisson-Boltzmann (NLPB) electrostatics [20-22] removes most of the Debye-Hückel approximations at greater computational expense. NLPB is a mean-field treatment and neglects ion-ion correlations [23,24] and ion size effects [25-27]. For monovalent ions where these correlations are weak, NLPB performs well, but it is less accurate for divalent $\mathrm{Mg}^{2+}[26,28]$. The tightly bound ion model $[24,29]$ accounts for ion-ion correlations and captures the ionic atmosphere well, but it is a Monte Carlo technique and has not yet been adapted for molecular dynamics. Manning counterion condensation theory $[7,30,31]$ can describe nonlinear effects near the RNA, but it is typically limited to low concentrations and linear or helical RNA geometry.

We recently developed a coarse-grained model with explicit $\mathrm{Mg}^{2+}$ and implicit $\mathrm{KCl}$ that revealed the importance of accounting for competition between $\mathrm{Mg}^{2+}$ and 
condensed $\mathrm{KCl}$ [32]. As a first approximation, $\mathrm{KCl}$ condensation was treated as a static function of $\mathrm{Mg}^{2+}$ concentration and fit to native basin experimental data. This approximation rendered the model valid only for native basin fluctuations of experimentally characterized RNA. A dynamic, physics-based description of $\mathrm{KCl}$ condensation is needed for the model to have any predictive power.

In this Letter, we introduce a generalized Manning counterion condensation model that describes folded RNA at physiological ionic concentrations. $\mathrm{Mg}^{2+}$ is treated explicitly to account for ion-ion correlations, while $\mathrm{KCl}$ condensation is described by the generalized Manning model. We add the electrostatic model to a coarse-grained model of RNA to capture native basin fluctuations. The coarse-grained model is an all heavy atom structure-based model [32-34] with a theoretical base in the energy landscape theory of protein folding [35-37]. The model is in good agreement with experimental measurements of the ion atmosphere within the native basin for several compact RNA molecules at varying $\mathrm{KCl}$ and $\mathrm{Mg}^{2+}$ concentrations. The model is also applicable beyond the native basin, and the implicit treatment of $\mathrm{KCl}$ makes the model computationally inexpensive.

Classical Manning counterion condensation [7] occurs on an infinite line of charge in the low concentration limit due to competition between mixing entropy and electrostatic energy. The mixing free energy per phosphate to condense $\theta$ ions per phosphate is given by

$$
G_{\text {Mix }}=k_{B} T \theta \ln \left(\frac{\theta}{e c V}\right),
$$

where $k_{B}$ is Boltzmann's constant, $T$ is the temperature, $c$ is the bulk concentration of the counterion, $V$ is the volume per phosphate into which the counterions condense, and $e$ is Euler's number. The condensed ions effectively rescale the charge of the phosphates by $1-z \theta$, so the electrostatic energy per phosphate is given by

$$
G_{E}=k_{B} T(1-z \theta)^{2} F,
$$

where $z$ is the charge of the counterion and $F$ is the DebyeHückel energy of the bare polyelectrolyte charges per phosphate in units of $k_{B} T$. For an infinite line of charge at low concentration, $G_{\text {Mix }}$ and $G_{E}$ (through $F$ ) both diverge like $\ln c$, so the condensation is constant over a wide range of counterion concentrations [7].

Condensation on RNA under physiological conditions differs from classical Manning condensation in two important regards that require the inclusion of additional physics. First, folded RNA is not a line of charge, so $F$ does not diverge like $\ln c$, and condensation is not constant over wide concentration ranges. Folded RNA rather forms compact and irregular structures, so a model allowing varying condensation on each phosphate is required to account for the electrostatic heterogeneity of the phosphates. This can be accomplished by making $F$ a dynamical function of phosphate coordinates and adding appropriate phosphate indices to Eqs. (1) and (2). Second, salts are present at intermediate concentrations. As a result, there is a large population of screening ions near the RNA in addition to the condensed ions that contribute to the mixing free energy. Many of these implicit screening ions occupy the ion inaccessible volume of the RNA and must be removed. Accounting for screening ions and ion inaccessible volume requires more substantial extensions to Manning counterion condensation, which we outline below. The resulting generalization of Manning counterion condensation may also have applications in polyelectrolyte theory beyond RNA.

The $\mathrm{K}^{+}$and $\mathrm{Cl}^{-}$distributions may be divided into screening ions and Manning condensed ions. The screening ion density is given by a linearized Poisson-Boltzmann distribution, while the condensed ion density captures deviations of the distribution from linearity near RNA. In Debye-Hückel theory, screening ions of species $s$ have a local density

$$
n_{\mathrm{DH}, s}(\vec{r})=c_{s}\left(1-\frac{z_{s} \Phi_{0}(\vec{r})}{k_{B} T}\right)
$$

that varies linearly with the electrostatic potential $\Phi_{0}$, where $c_{s}$ and $z_{s}$ are the concentration and charge of ionic species $s$. For strong potentials, nonphysical negative concentrations are possible, as frequently occurs for $\mathrm{Cl}^{-}$near RNA. These negative concentrations must be corrected by a corresponding positive concentration of condensed ions. Consequently, it is necessary to account for both condensed $\mathrm{K}^{+}$and $\mathrm{Cl}^{-}$to avoid negative local concentrations of $\mathrm{Cl}^{-}$.

In the present work, the density of Manning condensed ions is modeled as the sum of two normalized Gaussian distributions $P(r, \sigma)$ centered on the position of every RNA charge:

$$
\begin{aligned}
& n_{\mu, s}(\vec{r})=\sum_{i} \mu_{i s} P\left(\left|\vec{r}-\vec{r}_{i}\right|, \sigma_{\mu}\right), \\
& n_{\eta, s}(\vec{r})=\sum_{i} \eta_{i s} P\left(\left|\vec{r}-\vec{r}_{i}\right|, \sigma_{\eta}\right),
\end{aligned}
$$

with charges placed on every phosphate. The total density of ions is then $n_{\mathrm{DH}, s}+n_{\mu, s}+n_{\eta, s}$. The mixing Gaussian controls mixing free energy and the size $\sigma_{\mu}=0.7 \mathrm{~nm}$ is set to the Bjerrum length. The hole Gaussian enforces the ion accessibility by offsetting any ions too close to the RNA and the size $\sigma_{\eta}=0.34 \mathrm{~nm}$ is set approximately to the closest approach of a hydrated ion to RNA. The sensitivity of the results to the two free parameters $\sigma_{\mu}$ and $\sigma_{\eta}$ is shown in the Supplemental Material [38]. The Manning condensed ions of species $s$ at a charged atom $i$ are then given by $\theta_{i s}=\mu_{i s}+\eta_{i s}$. In the model, $\mathrm{K}^{+}$and $\mathrm{Cl}^{-}$only condense on phosphates or RNA charges, so $\mu_{i s}=\eta_{i s}=0$ at explicit $\mathrm{Mg}^{2+}$ charges. 
In contrast to the implicit $\mathrm{K}^{+}$and $\mathrm{Cl}^{-}$distributions, the $\mathrm{Mg}^{2+}$ distribution is determined by the location of the explicit ions. In addition to electrostatic forces, $\mathrm{Mg}^{2+}$ ions interact with an $r^{-12}$ excluded volume potential parametrized to keep each $\mathrm{Mg}^{2+}$ the fully hexahydrated distance from the RNA and other $\mathrm{Mg}^{2+}$ ions [32].

The electrostatic free energy of the RNA and the condensed ions can be given in terms of Debye-Hückel interactions

$$
\begin{gathered}
\phi\left(r_{i j}, 0\right)=k_{B} T \frac{l_{B}}{r_{i j}} \exp \left(-\kappa r_{i j}\right), \\
\phi\left(r_{i j}, \sigma\right)=k_{B} T \sum_{a= \pm 1} \frac{-1}{2} \frac{l_{B}}{a r_{i j}} \exp \left(\frac{1}{2} \kappa^{2} \sigma^{2}+\kappa a r_{i j}\right) \\
\times\left[1-\operatorname{erf}\left(\frac{a r_{i j}+\kappa \sigma^{2}}{\sigma \sqrt{2}}\right)\right],
\end{gathered}
$$

where $r_{i j}$ is the distance between particles $i$ and $j, \kappa$ is the inverse Debye length, and $l_{B}$ is the Bjerrum length (approximately $7 \AA$ in water at $300 \mathrm{~K}$ ). The interaction between two point charges is $\phi\left(r_{i j}, 0\right)$, while the interaction between a point and a Gaussian of variance $\sigma^{2}$ is $\phi\left(r_{i j}, \sigma\right)$, and the interaction between two Gaussians of variance $\sigma_{m}{ }^{2}$ and $\sigma_{n}{ }^{2}$ is $\phi\left(r_{i j}, \sqrt{\sigma_{m}^{2}+\sigma_{n}^{2}}\right)$. The total electrostatic free energy $G_{E}$ and electrostatic potential $\Phi$ are then

$$
\begin{gathered}
G_{E}=\frac{1}{2} \sum_{i j} \sum_{m n} q_{m, i} q_{n, j} \phi\left(r_{i j}, \sqrt{\sigma_{m}^{2}+\sigma_{n}^{2}}\right), \\
\Phi_{m}(\vec{r})=\sum_{j} \sum_{n} q_{n, j} \phi\left(\vec{r}-\vec{r}_{j}, \sqrt{\sigma_{m}^{2}+\sigma_{n}^{2}}\right),
\end{gathered}
$$

where the sum on indices $m$ and $n$ runs over the three labels $\{0, \mu, \eta\}$, denoting points, mixing Gaussians, and hole Gaussians, respectively; and the sum on $i$ and $j$ runs over all charged atoms including $i=j$ (except when $m=n=0$ ) so that condensed ions can interact with their own phosphate. For points, $\sigma_{0}=0 \mathrm{~nm}$, and $q_{0, i}$ denotes the charge of particle $i$, while the condensed ion charges are $q_{\mu, i}=$ $\sum_{s} z_{s} \mu_{i s}$ and $q_{\eta, i}=\sum_{s} z_{s} \eta_{i s}$. The actual potential is $\Phi_{0}$, while $\Phi_{\mu}$ and $\Phi_{\eta}$ are the average of the potential over the Gaussian regions.

At intermediate salt, the mixing free energy in Eq. (1) must be reformulated to include screening ions. The substitution $n=\theta / V$ allows the mixing free energy to be expressed in terms of local ion density and condensation volume. The local density $n_{\mathrm{Mix}, i s}$ can be approximated by averaging $n_{\mathrm{DH}, s}$ over the mixing Gaussian and adding $n_{\mu, s}$ :

$$
n_{\mathrm{Mix}, i s}=c_{s}\left(1-\frac{z_{s} \Phi_{\mu}\left(\vec{r}_{i}\right)}{k_{B} T}\right)+n_{\mu, s}\left(\vec{r}_{i}\right) .
$$

The effective volume a Gaussian occupies can be estimated as the inverse of the local Gaussian density

$$
V_{m, i}=1 / \sum_{j} P\left(r_{i j}, \sigma_{m}\right),
$$

where the sum on $j$ runs over all phosphates. The density of holes $n_{\eta, s}\left(\vec{r}_{i}\right)$ is omitted from Eq. (10) because this term serves primarily to make the ion density average to zero within the hole volume $V_{\eta, i}$; consequently, this empty volume is subtracted off the mixing volume $V_{\mu, i}$, giving $V_{\mu, i}-V_{\eta, i}$. The mixing free energy is then approximately

$$
G_{\mathrm{Mix}}=\sum_{i} \sum_{s} k_{B} T n_{\mathrm{Mix}, i s}\left(V_{\mu, i}-V_{\eta, i}\right) \ln \left(n_{\mathrm{Mix}, i s} / e c_{s}\right) .
$$

The potential ensures that $n_{\mathrm{Mix}, i s} \geq 0$, so any nonphysical negative concentration of screening ions will be balanced by a positive concentration of condensed ions.

The electrostatic and mixing free energy of the screening ions are typically ignored because they cancel each other out for weak potentials. Since the mixing free energy of the screening ions within the volume $V_{\mu, i}-V_{\eta, i}$ has been included in the free energy, the electrostatic free energy of the screening ions in this region must be included as well. This free energy is given by

$$
G_{E S}=\frac{1}{2} \sum_{i} \sum_{s} z_{s} c_{s}\left(1-\frac{z_{s} \Phi_{\mu}\left(\vec{r}_{i}\right)}{k_{B} T}\right)\left(V_{\mu, i}-V_{\eta, i}\right) \Phi_{\mu}\left(\vec{r}_{i}\right) .
$$

With generalizations for the electrostatic free energy $G_{E}$ and the mixing free energy $G_{\mathrm{Mix}}+G_{E S}$, it is necessary to enforce ion accessibility near the RNA. The concentration of each ionic species $n_{\mathrm{Hol}, \text { is }}$ within the excluded volume of polyelectrolyte particle $i$ is

$n_{\mathrm{Hole}, i s}=c_{s}\left(1-\frac{z_{s} \Phi_{\eta}\left(\vec{r}_{i}\right)}{k_{B} T}\right)+n_{\mu, s}\left(\vec{r}_{i}\right)+n_{\eta, s}\left(\vec{r}_{i}\right)$,

where the screening ions have been averaged over the hole Gaussian. Since ions are excluded from this volume, $n_{\mathrm{Hole}, i s}=0$. Rather than using constraints, a strong harmonic restraint

$$
G_{\text {Hole }}=\frac{1}{2} k_{\text {Hole }} \sum_{i} \sum_{s} n_{\text {Hole }, i s}^{2}
$$

is added to the potential to keep $\eta$ within 0.01 ions of the correct value. Furthermore, to maintain stability and avoid overfitting the ion distribution, $\mu_{i s}$ and $\eta_{i s}$ are weakly harmonically restrained to $n_{\mu, s}\left(\vec{r}_{i}\right) V_{\mu, i}$ and $n_{\eta, s}\left(\vec{r}_{i}\right) V_{\eta, i}$, respectively, by a term $G_{\text {Rest }}$. Together with the $\mathrm{Mg}^{2+}$ and RNA 
positions, the four condensation variables for each phosphate $\left(\mu_{i+}, \mu_{i-}, \eta_{i+}\right.$, and $\left.\eta_{i-}\right)$ are treated as coordinates that evolve by Langevin dynamics on the potential $G_{E}+G_{\text {Mix }}+$ $G_{E S}+G_{\text {Hole }}+G_{\text {Rest }}$. Parameter values and other simulation details may be found in the Supplemental Material [38].

The ion atmosphere can be quantified by the number of excess ions of each species which associate with an RNA. The number of excess ions of a particular ionic species varies with the concentration, but the total charge of all excess ions must balance the RNA charge. At fixed $\mathrm{KCl}$ concentration, with $c_{+} \gg c_{2+}$, the $\mathrm{Mg}^{2+}$-RNA interaction free energy

$$
\Delta G_{\mathrm{Mg}^{2+}}=-k_{B} T \int_{0}^{c_{2+}} \Gamma_{2+} d \ln c_{2+}^{\prime}
$$

is directly related to the excess $\mathrm{Mg}^{2+}, \Gamma_{2+}$, as a function of the $\mathrm{Mg}^{2+}$ concentration [48]. $\Gamma_{2+}$ can be measured experimentally with a fluorescent chelator $[15-17,48]$ or other techniques [49] and can be calculated from simulations $[32,50]$. Because $\Gamma_{2+}$ bridges between simulation and experiment and is directly related to the $\mathrm{Mg}^{2+}-\mathrm{RNA}$ interaction free energy, it is an ideal quantity for testing models of RNA electrostatics.

The model is able to reproduce $\Gamma_{2+}$ for the adenine riboswitch at $50 \mathrm{mM} \mathrm{KCl}$ and for a 58 nucleotide fragment of the ribosome at several $\mathrm{KCl}$ concentrations (Fig. 1).
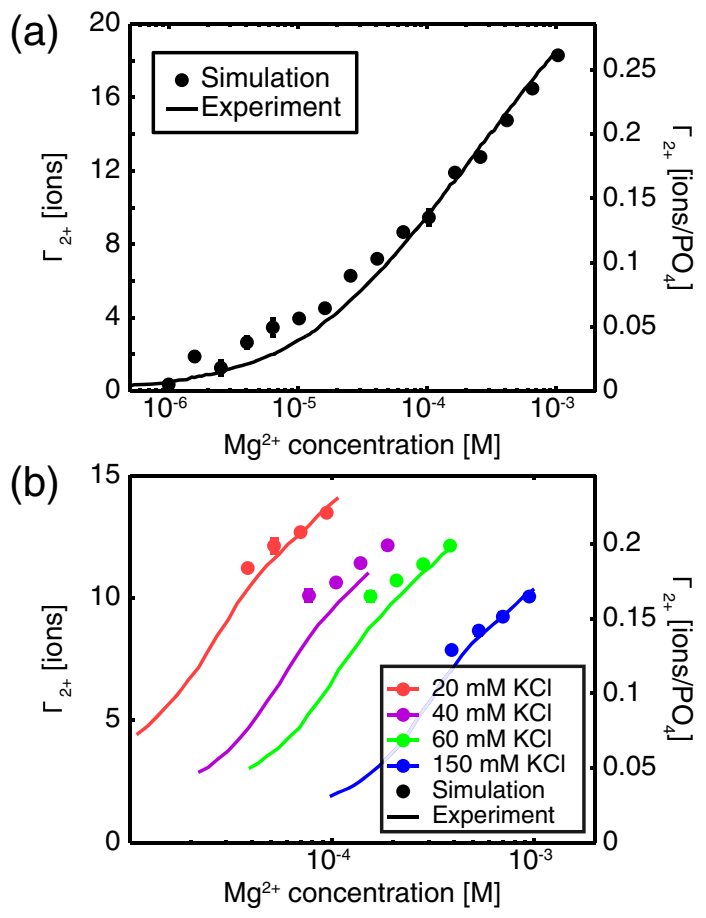

FIG. 1 (color online). The model captures excess $\mathrm{Mg}^{2+}$ over a wide range of concentrations for both (a) the adenine riboswitch at $50 \mathrm{mM} \mathrm{KCl}$, and (b) a 58 nucleotide ribosomal fragment. Experimental results are plotted as lines and simulation results are plotted as dots.
Experimental data for the adenine riboswitch and the ribosomal fragment are taken from Refs. [15,16], respectively. The transferability of the model to several $\mathrm{KCl}$ concentrations in Fig. 1(b) bolsters the model, as simpler models lacking RNA excluded volume in Eq. (15) can be fit at a single $\mathrm{KCl}$ concentration, but they break down when applied to multiple $\mathrm{KCl}$ concentrations (data not shown). Lower $\mathrm{Mg}^{2+}$ concentrations were not explored for the ribosomal fragment because the system undergoes a conformational change we did not wish to model near the inflection point in the experimental data. With further calibration of the underlying coarse-grained model, the generalized Manning condensation model could be used to probe the electrostatic effects driving the conformational transition. The excellent agreement over a wide range of $\mathrm{Mg}^{2+}$ and $\mathrm{KCl}$ concentrations suggests that the model is capturing the electrostatic free energy quite well.

Native basin fluctuations can have a notable effect on $\Gamma_{2+}$. For the beet western yellow virus pseudoknot the number of excess $\mathrm{Mg}^{2+}$ has been measured [17]. In the generalized Manning condensation model, the excess $\mathrm{Mg}^{2+}$ is overestimated if the RNA is frozen in the crystal structure, but it can be corrected by allowing the RNA to fluctuate (Fig. 2). This occurs because the crystal structure contains a negatively charged pocket between the $5^{\prime}$ triphosphate tail and the rest of the pseudoknot where $\mathrm{Mg}^{2+}$ binds nonspecifically. This pocket is not stable in solution due to phosphate-phosphate repulsion, resulting in accurate $\Gamma_{2+}$ predictions when the RNA dynamics are included. The sensitivity of $\Gamma_{2+}$ to small native basin fluctuations reveals the importance of modeling the full conformational ensemble to accurately predict $\Delta G_{\mathrm{Mg}^{2+}}$. Sensitivity has been previously observed in partially unfolded ensembles, which exhibit larger fluctuations $[16,17]$. Our model is ideally designed to capture these conformational ensembles.

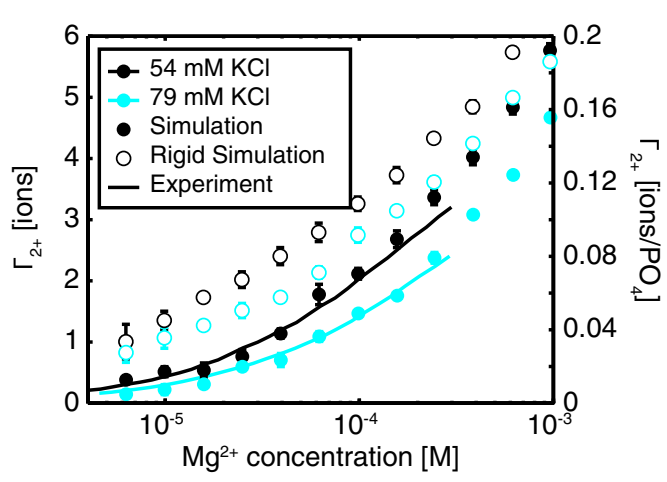

FIG. 2 (color online). Predictions by the model are too high for the beet western yellow virus pseudoknot in the rigid simulation where the RNA is fixed in the crystal structure (the open circles). The agreement with experiment [17] is quite close if native basin fluctuations are included (the solid circles). 
A comparison of the generalized Manning model, NLPB, and previous explicit solvent predictions [50] of $\Gamma_{2+}$ for the SAM-I riboswitch is shown in the Supplemental Material [38]. Explicit solvent simulations appear to underpredict $\Gamma_{2+}$, possibly because $\mathrm{K}^{+}$can dehydrate too easily [51] and drive away $\mathrm{Mg}^{2+}$. Many ion parameters have been proposed for explicit solvent simulation [52-55]. The excess $\mathrm{Mg}^{2+}$ is a sensitive measure of RNA electrostatics that may be useful in future calibration of explicit solvent ion parameters.

The generalized Manning model provides a more accurate description of the ion atmosphere than the conventional descriptions of Debye-Hückel, the nonlinear Poisson-Boltzmann equation, classical Manning counterion condensation, and possibly even explicit solvent simulation. This success is due in part to the explicit treatment of $\mathrm{Mg}^{2+}$ that accounts for ion-ion correlations [24] absent from mean-field treatments. The implicit treatment of $\mathrm{KCl}$ makes the model computationally inexpensive, both by significantly reducing the number of particles in the large boxes required for a bath of free $\mathrm{Mg}^{2+}$ and by allowing the use of the short range DebyeHückel potential rather than the long range Coulomb potential.

Our previous model of RNA electrostatics served as a valuable proof of concept: $\Gamma_{2+}$ can be predicted correctly by accounting for $\mathrm{KCl}$ condensation [32]. In that model, $\mathrm{KCl}$ condensation was treated as an experimentally fit function of $\mathrm{Mg}^{2+}$ concentration that was static within a simulation, but this approximation introduced extensive limitations that made the model untransferable and unusable for dynamics. The fit to experiment meant that the model could only be used on RNA systems at specific $\mathrm{KCl}$ concentrations where $\Gamma_{2+}$ had been measured. The assumption of static $\mathrm{KCl}$ condensation confined one to a single conformational basin where $\mathrm{KCl}$ condensation does not change and introduced inconsistencies that undermine Eq. (16).

The model presented in this Letter removes all of these limitations by making $\mathrm{KCl}$ condensation a dynamical quantity dependent on atomic coordinates and calculated from physical principles. Since the potential is in terms of atomic coordinates and $\mathrm{KCl}$ condensation, it is transferable, phosphate-phosphate repulsion is automatically included, and $\mathrm{KCl}$ condensation can respond to conformational changes, making the model applicable beyond the native basin. Consequently, this model provides a description of the electrostatic features of the full RNA free energy landscape, and it allows calculation of $\Delta \Delta G_{\mathrm{Mg}^{2+}}$ between conformational basins. The electrostatic description is in a dynamic context that can access long time scales inaccessible through other techniques and can connect with experiments.

See the Supplemental Material [38] for a comparison with explicit solvent simulations, parameter sensitivity analysis, further details of the model, and Refs. [39-47]. The parallel code used to simulate the potential is available for download [56].

Work at the Center for Theoretical Biological Physics was sponsored by the NSF (Grants No. PHY1427654 and No. MCB-1214457), by the NIH (Grant No. 1R01GM110310-01A1), and by the Welch Foundation (Grant No. C-1792). A. M. was supported by the NSF (Grant No. PHY-1216435). P. C. W. was supported by a NSF CAREER Award (Grant No. MCB-1350312). K. Y. S. acknowledges support by the National Institutes of Health (Grant No. 1R01GM110310-01A1). U.M. acknowledges support as a John Simon Guggenheim Memorial Foundation fellow. J. N. O. acknowledges support as a CPRIT Scholar in Cancer Research sponsored by the Cancer Prevention and Research Institute of Texas. Computing resources were provided by Rice University and supported in part by the Cyberinfrastructure for Computational Research funded by NSF under Grant No. CNS-0821727.

*Corresponding author. jonuchic@ rice.edu

[1] D. E. Draper, RNA 10, 335 (2004).

[2] C. Hyeon, N. A. Denesyuk, and D. Thirumalai, Isr. J. Chem. 54, 1358 (2014).

[3] J.-C. Lin and D. Thirumalai, J. Am. Chem. Soc. 135, 16641 (2013).

[4] P. C. Whitford, P. Geggier, R. B. Altman, S. C. Blanchard, J. N. Onuchic, and K. Y. Sanbonmatsu, RNA 16, 1196 (2010).

[5] B. Lutz, M. Faber, A. Verma, S. Klumpp, and A. Schug, Nucleic Acids Res. 42, 2687 (2014).

[6] A. M. Mustoe, H. M. Al-Hashimi, and C. L. Brooks III, J. Phys. Chem. B 118, 2615 (2014).

[7] G. S. Manning, Q. Rev. Biophys. 11, 179 (1978).

[8] D. Lambert, D. Leipply, R. Shiman, and D. E. Draper, J. Mol. Biol. 390, 791 (2009).

[9] E. Koculi, C. Hyeon, D. Thirumalai, and S. A. Woodson, J. Am. Chem. Soc. 129, 2676 (2007).

[10] S. L. Heilman-Miller, D. Thirumalai, and S. A. Woodson, J. Mol. Biol. 306, 1157 (2001).

[11] D. Thirumalai and C. Hyeon, in Non-Protein Coding RNAs, edited by N. G. Walter, S. A. Woodson, and R. T. Batey, Springer Series in Biophysics (Springer, Berlin, 2009), Chap. 2, p. 27.

[12] J. Wittmer, A. Johner, and J. F. Joanny, J. Phys. II (France) 5, 635 (1995).

[13] S. L. Heilman-Miller, J. Pan, D. Thirumalai, and S. A. Woodson, J. Mol. Biol. 309, 57 (2001).

[14] S. P. Hennelly, I. V. Novikova, and K. Y. Sanbonmatsu, Nucleic Acids Res. 41, 1922 (2013).

[15] D. Leipply and D. E. Draper, Biochemistry 50, 2790 (2011).

[16] D. Grilley, V. Misra, G. Caliskan, and D. E. Draper, Biochemistry 46, 10266 (2007). 
[17] A. M. Soto, V. Misra, and D. E. Draper, Biochemistry 46, 2973 (2007).

[18] C. Hyeon and D. Thirumalai, Proc. Natl. Acad. Sci. U.S.A. 102, 6789 (2005).

[19] S. S. Cho, D. L. Pincus, and D. Thirumalai, Proc. Natl. Acad. Sci. U.S.A. 106, 17349 (2009).

[20] B. Honig and A. Nicholls, Science 268, 1144 (1995).

[21] S. W. Chen and B. Honig, J. Phys. Chem. B 101, 9113 (1997).

[22] N. A. Baker, D. Sept, S. Joseph, M. J. Holst, and J. A. McCammon, Proc. Natl. Acad. Sci. U.S.A. 98, 10037 (2001).

[23] A. A. Chen, M. Marucho, N. A. Baker, and R. V. Pappu, in Biophysical, Chemical, and Functional Probes of RNA Structure, Interactions and Folding: Part B, Methods in Enzymology Vol. 469, edited by D. Herschlag (Academic Press, New York, 2009), p. 411.

[24] Z. He and S.-J. Chen, J. Chem. Theory Comput. 8, 2095 (2012).

[25] A. H. Boschitsch and P. V. Danilov, J. Comput. Chem. 33, 1152 (2012).

[26] V. B. Chu, Y. Bai, J. Lipfert, D. Herschlag, and S. Doniach, Biophys. J. 93, 3202 (2007).

[27] I. Borukhov and D. Andelman, Phys. Rev. Lett. 79, 435 (1997).

[28] J. Lipfert, S. Doniach, R. Das, and D. Herschlag, Annu. Rev. Biochem. 83, 813 (2014).

[29] G. Chena, Z.-J. Tana, and S.-J. Chen, Biophys. J. 98, 111 (2010).

[30] G. S. Manning, Biophys. Chem. 101-102, 461 (2002).

[31] N. A. Denesyuk and D. Thirumalai, J. Phys. Chem. B 117, 4901 (2013).

[32] R. L. Hayes, J. K. Noel, P. C. Whitford, U. Mohanty, K. Y. Sanbonmatsu, and J. N. Onuchic, Biophys. J. 106, 1508 (2014).

[33] P. C. Whitford, J. K. Noel, S. Gosavi, A. Schug, K. Y. Sanbonmatsu, and J. N. Onuchic, Proteins 75, 430 (2009).

[34] P. C. Whitford, A. Schug, J. Saunders, S. P. Hennelly, and J. N. Onuchic, Biophys. J. 96, L7 (2009).

[35] J. N. Onuchic, Z. Luthey-Schulten, and P. G. Wolynes, Annu. Rev. Phys. Chem. 48, 545 (1997).

[36] R. Zwanzig, A. Szabo, and B. Bagchi, Proc. Natl. Acad. Sci. U.S.A. 89, 20 (1992).

[37] J. D. Bryngelson and P. G. Wolynes, Proc. Natl. Acad. Sci. U.S.A. 84, 7524 (1987).
[38] See Supplemental Material at http://link.aps.org/ supplemental/10.1103/PhysRevLett.114.258105, which includes Refs. [39-47], for a comparison with explicit solvent simulations, parameter sensitivity analysis, and further details of the model.

[39] S. Kirmizialtin, A. R. Silalahi, R. Elber, and M. O. Fenley, Biophys. J. 102, 829 (2012).

[40] S. Kirmizialtin, S. A. Pabit, S. P. Meisburger, L. Pollack, and R. Elber, Biophys. J. 102, 819 (2012).

[41] A. Serganov, Y.-R. Yuan, O. Pikovskaya, A. Polonskaia, L. Malinina, A. T. Phan, C. Hobartner, R. Micura, R. R. Breaker, and D. J. Patel, Chem. Biol. 11, 1729 (2004).

[42] G. L. Conn, A. G. Gittis, E. E. Lattman, V. K. Misra, and D. E. Draper, J. Mol. Biol. 318, 963 (2002).

[43] L. Su, L. Chen, M. Egli, J. M. Berger, and A. Rich, Nat. Struct. Biol. 6, 285 (1999).

[44] R. K. Montange and R. T. Batey, Nature (London) 441, 1172 (2006).

[45] J. Wang, P. Cieplak, and P. A. Kollman, J. Comput. Chem. 21, 1049 (2000).

[46] W. F. Van Gunsteren and H. J. C. Berendsen, Mol. Simul. 1, 173 (1988).

[47] A. Savelyev and G. A. Papoian, J. Phys. Chem. B 112, 9135 (2008).

[48] D. Grilley, A. M. Soto, and D. E. Draper, Proc. Natl. Acad. Sci. U.S.A. 103, 14003 (2006).

[49] Y. Bai, M. Greenfeld, K. J. Travers, V. B. Chu, J. Lipfert, S. Doniach, and D. Herschlag, J. Am. Chem. Soc. 129, 14981 (2007).

[50] R. L. Hayes, J. K. Noel, U. Mohanty, P. C. Whitford, S. P. Hennelly, J. N. Onuchic, and K. Y. Sanbonmatsu, J. Am. Chem. Soc. 134, 12043 (2012).

[51] W. H. Braunlin, in Advances in Biophysical Chemistry, Vol. 5, edited by C. A. Bush (JAI Press, Greenwich, CT, 1995), p. 89.

[52] L. X. Dang, J. Am. Chem. Soc. 117, 6954 (1995).

[53] A. A. Chen and R. V. Pappu, J. Phys. Chem. B 111, 11884 (2007).

[54] O. Allnér, L. Nilsson, and A. Villa, J. Chem. Theory Comput. 8, 1493 (2012).

[55] A. Saxena and A. E. García, J. Phys. Chem. B 119, 219 (2015).

[56] See http://smog.rice.edu/SBMextension.html. 